\title{
Analysis of Costs and Benefits of Cloud Computing
}

\section{$\mathrm{PhD}$ candidate Albana Madhi (Kacollja)}

Lecturer in Faculty of Economy Elbasan

\author{
Prof.As. Dr. Gjergji Shqau
}

Lecturer in Faculty of Economy Elbasan

\author{
Aldi Lekaj \\ B.Sc. in Computer Engineering, MBA , Lysaker - Oslo Area
}

Doi:10.5901/ajis.2014.v3n3p259

\begin{abstract}
This article incorporating a theoretical and practical approach to cost-benefit analysis to help the managerial level of a company that is in front of a decision if or not to adopt Cloud Computing to the bussiness. Cost-benefit analysis is divided into six distinct sections to determine what costs and benefits are economically significant for the case study analyzed. Based on the above allocation sections TEI (Total Economic Impact) is applied, implemented by Forrester as a methodology. This methodology was chosen because it gives a general view of the impact and benefits in information technology to cloud computing adoption not only through the analysis of costs and benefits but also giving appropriate weight to information technology resources that are possessed. The article deals with analysis, forecasts and implementation of Cloud Computing. Showing financial benefits acquired from "green IT" as a consequence from this technology. Through a detailed analysis of the cloud computing phenomenon and its connection with green IT, this article conducts a cost-benefit analysis as a case study taking a internet and comunications services company, such as Primo Communications sh.p.k. The aim of the project is to show the potential benefits of the transition to cloud computing of this company, benefits expressed in increased efficiency of the use of technological resources or even increased environmental benefits. Together "cloud computing" and "green IT" treatment in this article represent two of the most challenging opportunities in business development nowadays.
\end{abstract}

\section{Approach and Methodology}

Forrester takes an approach of few more steps to assess the impact of cloud computing in an organization. Specifically, we followed these steps :

1. Interviewed staff of marketing, sales and product managers. Also human resources managers.

2. Interviewed 13 PRIMO Communications engineers working in various aspects of the organization's activity.

3. Built an orgasation model based on features extracted from the interviews.

4. Built a financial model using the TEI method. Financial model was populated with data about the costs and benefits that emerged after interviews.

5. Following the methodology, we analyze four essential elements:

- Cost

- Benefits to the entire organization

- Strategic Opportunities for flexibility

- The risk

Since large undertakings have a sophisticated investment analysis, particularly with regard to IT, TEI methodology serves the purpose for having a picture of the whole economic impact.

\section{The Purpose of this Article}

Cost-benefit analysis is done for one of the leading companies in providing 3N1 currently in our country, PRIMO Communications sh.p.k.

The project examines the fact that what will happen in the cost-benefit point of view in the case of transition services this company from on-site (Situated in the flats of the company) compared with use of Amazon cloud services 
(AWS, Amazon Web Services) for the data center that she possesses.

\section{Important Information}

According to interviews done PRIMO Communications sh.pk staff, and the fact that we mention longer recognitions with this process and an author of the article has worked directly in these operations. We have built a general picture for the Total Economic Impact (TIE), of this change from on-site data center to Amazon cloud service. The hypothesis put forth by us is that benefits of the shift to cloud computing data center will reduce the total operational cost of keeping a data center on-site from the company said. Should mention the fact that many of the benefits of this transition are not easily translated into numerical values and thus we return on investment (ROI, Return On Investment).

However there are three main areas where operational costs will undergo reduction.

1. Cost savings of hardware devices as a result of using a lower level of computers AWS

2. Savings from energy consumption that is at least based on the loan of equipment to AWS

3. Savings in staff costs as a result of it the use of a large efficiency with solutions to problems.

In the end we can add engineering staff productivity will increase due to a higher efficiency of systems and time management as well as their best, all these follow the life cycle of a business with structured and productive. Translated to increase the value and quality of products and services offered that will generate increased consumer satisfaction. $4.6 \%$ risk.

At the end of the financial analysis for a period extending over three years'll see the total benefit is corrected by

Although the results of cost-benefit analysis were positive, environmental benefits are difficult to define and even more clearly in the numerical value to determine if they play a decisive role in the choice of cloud computing from this company. This relates to the fact that many of the biggest providers of cloud computing have been building huge data center and use energy in order to increase their effectiveness and do not take into account that are destroying the renewable energy or clean, this is also for the fact that no subsidy in the case of taking into account the protection of the environment. Secondly, the current legislation in force including the developed countries of the most experienced in this field do not give priority to reducing costs in the event of environmental protection or the reverse in the case of punishment for not taking basic influence in this direction.

\section{Motivations}

"Cloud computing" and "green IT" are two of the topics of discussions of unfamiliar business environments simultaneously in this period.

- Cloud computing is presented as a "revolution" in the information technology industry, fundamentally changing the way of doing business from a data center on-site in a cloud service based entirely on Internet.

- To Understand where cloud computing will impact on the protection of global warming on the basis of its impact on the environment by reducing the carbon footprint you leave providers of information technology services.

- Benefiting from the business and expanding the use of cloud computing

\section{Project Description}

Cost-benefit analysis in the case of cloud computing transition has been taken specifically for the company PRIMO Communications sh.pk The project analyzes the positive sides but also the risks that threaten change in this case if the company in question would use the services offered by Amazon

The benefits of cloud computing in the perspective of an ISP (Internet Provider srvice) since Internet is the basis of the functioning of this new variety from traditional to be seen from another angle. PRIMO Communications is a provider of "raw material" under this innovation in information technology, so, it is very supportive of this type of service.

PRIMO Communications sh.pk, currently offers a package of services in the market Trio, Internet, Phone and TV. Taking into account that the number of users of these services is always growing translates into the fact that this kind of business there is constant need for capacity to offer a quality and consistency in what they offer. 


\section{Action Plan}

The action plan for the transition to the cloud is organized as follows.

- Analyses will be supported on Amazon Web Services (AWS)

- Source for the transition to the cloud will be built on VPC (Virtual Private Cloud Amazon)

- IT staff will create a template Amazon Machine Image (AMI) for operating systems that is currently in use (middleware, libraries, relevant configuration data)

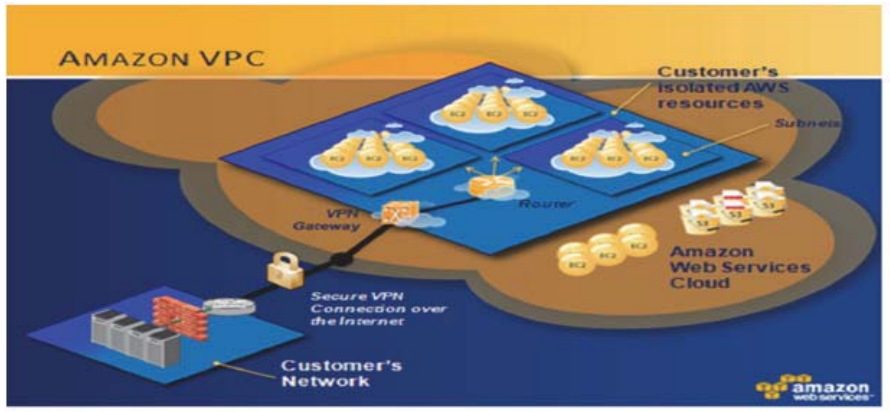

Figure 3: Skema e Amazon VPC

\section{Benefits of Cloud Technology}

Main economic benefit of technology cloud comes from the type of payment. This is a kind of payment, based on use, usually known as the conversion of capital costs (CAPEX) to operating costs (OPEX). The payment model we use differs from the usual rent, because the latter is a charge made for a certain period of use of computer resources in this case even though they can not be used at this time. Meanwhile cloud technology was spun for this payment is done only in case of exploitation of the resources required. Based payment model we use is completely independent of the period of use but appears only in case of use of those resources to customer needs. Finally, information technology analysts and its practitioners have concluded that there are some of the key forces that will affect the benefit in case of adoption of cloud technology.

\section{Financial Benefits}

Buying a computer or an application capacity (SaaS) delivered as a cloud service technology known as operating expense (OPEX) which may increase or decrease depending on the consumators variation request during an interval of time. The same solution but in a data center in an organization is a sunk cost which is known as capital cost (CAPEX) and presented in the balance sheet as assets and amortized over the longer term time.

Transformation of capital costs in operating expenses of making financial decisions easier and less riskier to deal. Finally, are summarized in a table financial benefits between CAPEX and OPEX.

\section{Comparison CAPEX and OPEX}

\begin{tabular}{lll}
\hline \multicolumn{1}{c}{ Factor } & \multicolumn{1}{c}{ Traditional Technology } & \multicolumn{1}{c}{ Cloud Technology } \\
\hline 1-Type of expense & Capital Expense (CAPEX) & Just (OPEX) \\
2- The flow of cash & Operational Expenses (OPEX) & Payments for offered service \\
3-Income & Maintenance Expenses & Maintenance payments \\
& Amortization of capital expenditures & Not recorded in the balance \\
4-Balance & Aktivet Afatgjata & 1-Hardware \\
& 1-Hardware & 2-Software \\
\hline
\end{tabular}




\section{Cloud Computing Costs}

In the preceding part explained the reasons why a firm is managed in cloud technology.

We will address the following differences in the total cost (TCO) by confronting cloud computing and the construction of a data center specific for a business. One for a comparison between cloud computing and data center is very difficult because of the hidden and indirect costs that appear in a data center firm (in-house).

There are many arguments and counterarguments regarding the total cost (TCO) between the two types of cloud computing technology and in-house because the costs are very specific and depend on the type of business, companies anyway to type in information technology or support their activities web will certainly have a lower total cost in the case of cloud computing than in-house computing.

\section{Financial Analysis}

Tables that cash flows (cash flow) that are used in financial analysis starting with an initial investment column reflecting costs incurred at time 0 or at the beginning of year 1. These costs are not discounted. Calculations for the present value (PV) were performed for each total cost and benefit. Present value calculations are shown in the table are summarized and the amount of inflows and outflows, with a discount rate every year. Table lists the main assumptions used for measurements in this chapter.

The table below provides approximate estimates of rates of annual use of datacenter processing nodes during the year. A use 100\% means that the server using 8736 hours each year. Measured this by 24 hours * 7 days * 52 weeks. Some types can not be shared servers in use which means that their use is only intended for a single user. These are mainly small computers. Shared servers can be used by several users at the same time.

The Percentages of Annual Use of Processing Nodes Dc

\begin{tabular}{|c|c|c|c|c|c|}
\hline Ref & $\begin{array}{l}\text { Tye of } \\
\text { Servers }\end{array}$ & Typical Tasks & Typical Usage Model & $\begin{array}{c}\text { Number of } \\
\text { hours per year }\end{array}$ & $\begin{array}{c}\text { Annual } \\
\text { Percentage of Use }\end{array}$ \\
\hline A11 & Small & For the daily office tasks & 8 hrs * 5 days * 47 weeks & 1880 ore & $21 \%$ \\
\hline A12 & Small & For software testing puproses & 8 hrs * 5 days * 47 weeks & 1880 ore & $21 \%$ \\
\hline A13 & Medium & For different customers & 24 hrs * 7 days * 52 weeks & 8736 ore & $100 \%$ \\
\hline A14 & Big & $\begin{array}{l}\text { For larger software development, } \\
\text { documentation }\end{array}$ & 10 hrs * 5 days * 47 weeks & 2350 ore & $27 \%$ \\
\hline A15 & Very Big & $\begin{array}{l}\text { Servers for the main services in the } \\
\text { company }\end{array}$ & $24 \mathrm{hrs}$ * 7 days * 52 weeks & 8736 ore & $100 \%$ \\
\hline
\end{tabular}

\section{Costs for the Staff}

When companies own and operate servers in their offices they need to hire full-time personal to select, acquire, set, supported and managed them. Server administrators perform a variety of tasks, including: updating the server, returns, movement, incident management and problem management and monitoring of salespeople. Table 13 describes the assumptions for staff salary is charged to assist the datacenter ongoing operation. Table 14 gives a forecast of annual salary for this staff. Staff who will assist consists of IT manager, two system administrators and 4 developers whose activity constitutes only $20 \%$ datacenter to day work.

The Annual Cost of Staff Salaries

\begin{tabular}{clcccc}
\hline Ref & \multicolumn{1}{c}{ Measurement Unit } & Basic Cost & Number & Decreasing rate & Salary \\
\hline B4 & IT manager`s Salary & 140000 & 1 & $100 \%$ & 140000 \\
B5 & System administrator's Salary & 110000 & 2 & $100 \%$ & 220000 \\
B6 & Developer's salary & 70000 & 4 & $20 \%$ & 56000 \\
B7 & TOTAL & & & & 416000 \\
\hline
\end{tabular}

\section{The Costs of the Datacenter in the Company}

Data center consists of only one main equipment room about $60 \mathrm{~m} 2$ and location of personnel by about $40 \mathrm{~m} 2$. We only 
included cost estimates are more visible and important. While ancillary costs such as: architecture costs, cabling, real estate companies, the building management, security datacenterit etc. are not very easily seen from everybody and determinable. Thus maintaining and operating an infrastructure includes the cost datacenteri other than those listed below, but we will assume that the following proposed assessment is reliable enough.

\section{Number of the Computers}

\begin{tabular}{|c|c|c|c|}
\hline $\begin{array}{l}\text { Type of } \\
\text { server }\end{array}$ & Hardware Specifications & Equivalent to AWS specifications & $\begin{array}{l}\text { Number of the } \\
\text { Computers }\end{array}$ \\
\hline $\begin{array}{l}\text { Small } \\
\text { Server }\end{array}$ & $\begin{array}{l}\text { Platform 32-bit, } 2 \text { GHz CPU, } 2 \text { GB } \\
\text { memory , } 160 \text { GB disc }\end{array}$ & $\begin{array}{l}\text { Small Instance } 1.7 \text { GB of memory, } 1 \text { EC2 Compute Unit (1virtual } \\
\text { core with } 1 \text { EC2 ComputeUnit), } 160 \text { GB storage, 32-bit platform }\end{array}$ & 3 \\
\hline $\begin{array}{l}\text { Meduim } \\
\text { Server }\end{array}$ & $\begin{array}{l}\text { Platform 64-bit, } 1 \text { quad-core } 2 \mathrm{GHz} \\
\mathrm{CPU} \text {, memory } 8 \mathrm{G} \text { RAM,500 GB } \\
\text { disc }\end{array}$ & $\begin{array}{l}\text { Large Instance } 7.5 \text { GB of memory, } 4 \text { EC2 Compute Units (2virtual } \\
\text { cores with } 2 \text { EC2 ComputeUnits each), } 850 \text { GB storage, 64-bit } \\
\text { platform }\end{array}$ & 5 \\
\hline Big Server & $\begin{array}{l}\text { Platform 64-bit, } 2 \text { quad-core } 2 \mathrm{GHz} \\
\text { CPUs, Memory } 15 \mathrm{~GB}, 850 \mathrm{~GB} \\
\text { disc }\end{array}$ & $\begin{array}{l}\text { Extra Large Instance } 15 \text { GB of memory, } 8 \text { EC2 Compute Units } \\
\text { (4virtual cores with } 2 \text { EC2 ComputeUnits each), } 1690 \text { GB } \\
\text { storage, 64-bit platform }\end{array}$ & 6 \\
\hline $\begin{array}{l}\text { Huge } \\
\text { Server }\end{array}$ & $\begin{array}{l}\text { Platform 64-bit, } 4 \text { quad-core } 2 \mathrm{GHz} \\
\text { CPUs, Memory } 32 \text { GB,1 TB disk }\end{array}$ & $\begin{array}{l}\text { High-Memory Double ExtraLarge Instance } 34.2 \text { GB of memory, } \\
13 \text { EC2 Compute Units( } 4 \text { virtual cores), } 850 \text { GB of local instance } \\
\text { storage, 64-bit platform }\end{array}$ & 4 \\
\hline Totali & & & 18 \\
\hline
\end{tabular}

Cost of hardware based on the total cost of acquisition, operation and substrate which comprises the datacenter servers, network equipment and hardware maintenance. Costs that include operating system software and application licenses and lease of the building costs are not calculated the financial analysis. This is because these costs are unchanged in terms of passing the assets of the company in the Amazon Cloud. When calculating the financial impact of datacenterit should consider the cost of replacing computers that is included in depreciation costs. As such, the initial cost of buying the hardware will be amortized over the useful life of the asset. In this case it is about 3 years (A4). Therefore using a fair depreciation expenses servers are minus the initial purchase at the end of life of dividing this value received for life. For simplicity, we will assume a value equal to zero rescue. Consequently, the annual cost of servers is calculated as follows:

Annual Server Cost $=\left(\right.$ Total number of servers ${ }^{*}$ Cost per Server $) /$ A4

Network Equipment Cost

Servers allow network devices to connect to each other and to the Internet. Network devices include firewalls, routers, switches, and intrusion detection systems and other equipment. We have assumed costs for equipment equal to $20 \%$ (A5) of the original value of the cost of amortizing the right server for three years of useful life (A4). The annual costs of network equipment is calculated by:

Annual cost of network equipment $=($ Total Cost per server * server * A5) $/$ A4

\section{Equipment Maintenance Cost}

Servers and network devices typically purchased with an annual contract maintenance for equipment repair in case of hardware defects. The annual maintenance cost is expressed as a percentage of the original purchase cost of servers and network devices that have parashikuarm first be approximately 10\% (A6). The annual cost of maintenance for servers and network equipment is calculated as follows:

- Annual cost of hardware maintenance (Hardware Annual Maintenance Cost) $=$ annual cost of maintenance of the servers (Annual Maintenance Cost of Servers) + annual maintenance cost of the network equipment (AnnualMaintenance Cost of Network Hardware), where:

- Annual cost of maintenance of servers $=$ total number of servers * Cost per server * $A 6$

- Annual cost of maintenance of network equipment $=$ Total Cost per server * server * $A 5 * A 6$

Servers operating costs and energy costs for cooling the servers

Datacenter servers not only consume energy, but also transform the latter into heat which must be removed from datacenteri to avoid over heating equipment. The power required to cool the servers datacenterit can agree or pass power consuming servers themselves. From a study carried out by the Green Grid estimated that most datacenter PUE have a value between 1.3 and 3.0. In this calculation we assumed a PUE of 2.5 (A3) as a conservative value. The reality may be even worse than this value because few measures are taken for increasing efficiency. Following equation was used to calculate the full power of estimating the energy demand of server multiplying it with PUE. Total energy use then 
multiplied by the average cost of electricity per kW / hour to assess the total annual cost of power. The annual costs for operation of servers and their cooling is calculated with the following formula:

- Annual cost of power and cooling (Annual Cost of Power and Cooling) $=$ total number of servers * standard energy consumption per server * $A 10$ * Annual Hours of work per server * $A 3$ * $(A 9 / 1000)$

The table gives an estimate of the annual costs associated maintenance and operation of the PRIMO Communications datacenter.

The Annual Cost of Operating and Maintaining the Dc in Primo Communications

\begin{tabular}{|c|c|c|c|c|c|c|c|}
\hline Ref & Item & Cost per Unit & Number of Units & Basic Cost & Year 1 & Year 2 & Year 3 \\
\hline \multicolumn{8}{|c|}{ Datacenter Servers Cost } \\
\hline $\mathrm{C} 1$ & Small Servers & 41000 & 3 & 123000 & 41000 & 41000 & 41000 \\
\hline $\mathrm{C} 2$ & Medium Servers & 145000 & 5 & 725000 & 241667 & 241667 & 241667 \\
\hline $\mathrm{C} 3$ & Big Servers & 295000 & 6 & 1770000 & 590000 & 590000 & 590000 \\
\hline $\mathrm{C} 4$ & Huge Servers & 597000 & 4 & 2388000 & 796000 & 796000 & 796000 \\
\hline \multicolumn{8}{|c|}{ Datacenter Equipment Costs } \\
\hline $\mathrm{C} 5$ & Network Equipment & & & 1001200 & 333733 & 333733 & 333733 \\
\hline \multicolumn{8}{|c|}{ Hardware maintenance cost of the datacenter } \\
\hline $\mathrm{C} 6$ & Hardware Maintenance & & & & 33373 & 33373 & 33373 \\
\hline $\mathrm{C} 7$ & Total Hardware Cost & & & 6007200 & 2035773 & 2035773 & 2035773 \\
\hline \multicolumn{8}{|c|}{ Switching on and Cooling Costs of the datacenter } \\
\hline $\mathrm{C} 8$ & Cooling (kW) & 12 lek/kW & 512000 & & 2048000 & 2048000 & 2048000 \\
\hline $\mathrm{C9}$ & Total cost of the datacenter & & & & 4083773 lek & 4083773 lek & 4083773 lek \\
\hline
\end{tabular}

\section{Summary of Basic Business Costs}

Adding annual staff cost to that datacenter costs take an assessment of actual costs for datacenterin business within the company PRIMO Communications.

Actual Costs

\begin{tabular}{clccc}
\hline Ref & \multicolumn{1}{c}{ Item } & Year 1 & Year 2 & Year 3 \\
\hline D1 & Staff Total Costs(B7) & 416000 & 436800 & 458640 \\
D2 & Datacenter Total Costs(C9) & 4083773 & 4083773 & 4083773 \\
D3 & Basic Total Costs & 4499773 lek & 4520573 lek & 4542413 lek \\
\hline
\end{tabular}

\section{Project Implementation Costs}

\section{1 phase costs of planning and design}

Less time will be needed for project planning, understanding of AWS technology, necessary design changes and in particular the design of modifications to be performed in virtual portal application. This portal is an application was designed and built within PRIMO Communications to facilitate the provisioning of virtual machines. This application needs to be modified in order to enable the creation and deployment of instances and volumes (EBS) in the AWS cloud. Need to develop additional functionality to support the rights of access control and automatic shutdown of computer resources which are not used. No external professional services will not be required to assist in the design and implementation of the project. Engineers working in PRIMO Communications are virtualization experts and so the manager in charge of this project should not have problems to find appropriate resources to properly design the project. However, the project will require a part time from other departments as networking and software engineers to give a more accurate solution. It is anticipated that efforts to become family with AWS technology and design of the solution will require about 20 days. Some people will be involved during this phase. The average full salary workers likely to be involved in the planning and designing of the project is around $46400 \mathrm{ALL}$ daily. 
Costs of Planning and Design Phase, Without Risk Adjustment

\begin{tabular}{clcc}
\hline Ref & Unit & Calculation & Beginner \\
\hline E1 & Work day & $((B 1+B 2+B 3) / 3) / A 7$ & 20 \\
E2 & Cost of daily charge & E1 * E2 & 46400 \\
E3 & Planning and design costs & 928000 \\
\hline
\end{tabular}

\subsection{Costs of implementing the project}

Implmentation phase of the project will be determined about 5 months which included the transfer of servers .At the initial stages and those that will follow it will require sufficient time for engineers to make the transition (migrate) the development of their existing environment to environment application using the AWS requires effort virtual.Kjo time equal to 2 days per server. So a total of 36 days. For ease of calculation will be used to calculate the value of pages in the preceding paragraph

\section{Cost of the Project Implementation, Without Risk Adjustment}

\begin{tabular}{clcc}
\hline Ref & Unit & Calculation & Beginner \\
\hline F1 & Work day & $((B 1+B 2+B 3) / 3) / A 7$ & 30 \\
F2 & Cost of daily charge & F1 * F2 & 46400 \\
F3 & Planning and design costs & 1392000 \\
\hline
\end{tabular}

\subsection{Cloud infrastructure costs}

In order to have an accurate financial analysis should determine the exact number of servers that will be transferred to the AWS and the number of those who stay in the existing datacenterin. Billed instances depending on the use of their ore. Price for each hour varies by type of instance and by geographical location. Amazon offers several possibilities for the manner of payment, as follows:

- Payment on demand using hourly rates / use

- Payment of annual simultaneous or more years for an instance

Therefore, to determine the number of instances you need and how long they need is the first step towards assessing the annual costs of AWS instances. For this purpose are defined two categories of instances to be provisioned to meet the demands for resources during the year.

- Baseline instances supposedly used by engineers for a period of the year. It represents the minimum level of demand for computer resources. Buying these instances will be based reservation model instances for a period of 3 years which match the amortization period and the period of analysis.

- Peak instance that is supposed to be used for a very short period of the year. They represent additional resources needed to meet the requirements in certain periods of the tests or by following the life cycle of different products. Buying such instances load model will be based on the prices of demand instances.

Annual Usage of instances per hour base load and is calculated as:

Annual Usage of instances per hour base load and is calculated as:

- Hours of use of ground instances (Hours of Baseline Instance Usage) = Number of instances * basic * Hours per year the average annual use of ground instances (Average Annual Usage of Baseline Instance)

- Hours of use uploaded instances (Hours of Peak Instance Usage) $=$ Number of instances * charged * Hours per year the average annual use charge instances (AverageAnnual Usage of Peak Instance)

The table below provides a summary of all costs Cloud solution.

Cloud Infrastructure without Risk Adjusment

\begin{tabular}{llcccccc}
\hline Ref & \multicolumn{1}{c}{ Units } & Cost per unit & Number of Units & Basic Cost & Year 1 & Year 2 & Year 3 \\
\hline & & Baseline & Instance based on AWS hourly usage / rate \\
J1 & Small Server & 3.9 lek & 5242 & 11925 & 20448 & 20448 \\
J2 & Medium Server & 16.1 lek & 8387 & 78764 & 135030 & 135030 \\
J3 & Big Server & 30.1 lek & 34944 & 613523 & 1051814 & 1051814 \\
J4 & Huge Server & 50.2 lek & 17472 & 511609 & 877094 & 877094 \\
\end{tabular}




\begin{tabular}{clccccc}
\hline J5 & Small Server & 8.1 lek & 0 & 6227 & 10675 & 10675 \\
J6 & Medium Server & 30.5 lek & 350 & 86734 & 148677 & 148677 \\
J7 & Big Server & 70.9 lek & 2097 & 66376 & 113795 & 113795 \\
J8 & Huge Server & 130.2 lek & 874 & & & \\
& & & & 23532 & 40344 & 40344 \\
J9 & Volume EBS (GB) & 8.9 & 4533 & 43016 & 73745 & 73745 \\
J10 & EBS IOPS in Million & 8.9 & 8286 & 1441706 & 2471637 & 2471637 \\
J11 & Total Cost of Cloud & & & & & \\
\hline
\end{tabular}

One should note that in the first year base and charged instances of AWS adjusted by subtracting a factor equal to 0.5833 to take into account the monthly payments towards the Amazon during the implementation which are not all at the same time.

\section{4 staff costs}

Since there will be a lot less physical severs to maintain the existing datacenter and since each engineer will be able to provide authority previously configured automatically via the virtual application, thought loads of staff datacenter management will be reduced significantly especially for both systems administrators. Table 23 below provides a recalculation of their annual salary based on the assumption that system administrators will spend less time on operations and will have free time to focus on other activities.

Recalculation of Annual Salary

\begin{tabular}{cccccc}
\hline Ref & \multicolumn{1}{c}{ Measurement Unit } & Basic Costs & Number & Decrease Rate & Salary \\
\hline K1 & IT manager salary & 140000 & 1 & $100 \%$ & 140000 \\
K2 & System administrator salary & 110000 & 2 & $80 \%$ & 176000 \\
K3 & Developers Salary & 70000 & 4 & $10 \%$ & 28000 \\
K4 & TOTAL & & & 344000 \\
\hline
\end{tabular}

\subsection{Total project costs of hybrid cloud}

Table 24 summarizes the overall project costs associated with the design, implementation, construction of infrastructure and staff salaries by $5 \%$ annual growth. The present value (PV) is calculated as follows:

$\mathrm{PV}=$ initial cost + Cost of Year $1 * .9091+.8264$ cost Vitit2 $*+{ }^{*} .7513$ Cost Vitit3

Summary of Total Costs of the Project

\begin{tabular}{clcccccc}
\hline Ref & \multicolumn{1}{c}{ Units } & Starting Cost & Year1 & Year 2 & Year 3 & Total & Actual Cost \\
\hline L1 & Planning and project Cost (E3) & 928000 & & & & 928000 & 928000 \\
L2 & Implementation Costs (F3) & 1392000 & & & & 1392000 & 1392000 \\
L3 & Staff Costs (K4) & & 344000 & 361200 & 379260 & 1084460 & 896164 \\
L4 & Cloud Infrastructure Costs (J11) & & 1441706 & 2471637 & 2471637 & 6384980 & 5210157 \\
L5 & Total costs of the project & 2320000 & 1785706 & 2832837 & 2850897 & 9789440 & 8426321 \\
\hline
\end{tabular}

\section{Benefits and Opportunities for Savings}

Most of the benefits that can be thought of in this project are not easily measurable in terms of ROI. The hypothesis is that the benefits of cloud computing should obviously reduce the overall costs of operations performed in PRIMO Communications. The purpose of this section is to express these benefits in money so we can put a monetary value to their effects. Some calculations made in this section shall be conducted under uncertainty. Thus we have two kinds of benefits:

- quantitative benefits that go directly to the calculation of ROI.

- qualitative benefits that are not directly involved in calculating ROI

However, qualitative benefits are just as important as quantitative and should be considered in the final evaluation of the project. Company the following assumptions concerning the assessment of benefits are accomplished by following categorization of benefits according to TEl. 


\subsection{Benefit from income}

Engineering activities and services PRIMO Communications bring income from the sale of products and services. The objective of this project is to cut costs where possible while staying within the budget that is allocated. So what is interesting in the category of benefits by income reductions are operational costs that can be achieved by the use of outside resources PRIMO Communications. As shown in Table 25 are two areas in which reductions can be achieved major costs:

- Reductions to electricity costs as a result of the passage of many servers in the Amazon cloud.

- Reductions in staff salary costs as a result of the greater effectiveness of the solution.

\section{Operational Benefits without Risk Adjustments}

\begin{tabular}{clccccc}
\hline Ref & \multicolumn{1}{c}{ Units } & Calculation & Year1 & Year2 & Year3 & Total \\
\hline M1 & Benefits from Electricity Power & C8- & 2048000 & 2048000 & 2048000 & 6144000 \\
M2 & Benefits from paying staff salary & D1-L3 & 72000 & 75600 & 79380 & 226980 \\
M3 & Total operational benefits & & 2120000 & 2123600 & 2127380 & 6370980 \\
\hline
\end{tabular}

Key Financial Indicators: Financial indicators, with risk adjustment

\begin{tabular}{lcccccccc}
\hline & Start & Year1 & Year2 & Year3 & IRR & Payback(vite) & ROI & NPV \\
\hline Costs & -2320000 & -1785706 & -2832837 & -2850897 & & & & \\
Benefits & & 3294667 & 3298267 & 3302047 & & & & \\
Savings & -2320000 & 1508961 & 465430 & 451150 & $3.5 \%$ & 3 & $4.6 \%$ & $503,508.05$ \\
\hline
\end{tabular}

The internal rate of return (IRR) of an indicator used in finance to compare odds from the investment. It is calculated as below:

- KursimetViti1 / (1 + IRR) + KursimetViti2 $1 /(1+$ IRR $) 2+$ KursimetViti3 $(1+$ IRR $) 3$ = initial investment

Return on Investment (ROI) for the three-year period is calculated:

- $\quad \mathrm{ROI}=(($ Initial Investment Gains- $) /$ initial investment $)$ * 100

Payback is the period that the company should take the return from the initial investment, ie to refund the amount of the initial invetimit., This indicator is expressed in years. Calculate returns based on savings rather than benefits.

Savings Year $1+$ Year $2+$ Savings Year 3> = Initial investment

NPV is the best indicator for decision making will be resolved if a project or not. It is calculated:

- $\mathrm{NPV}=$ Savings Year1 / $(1+$ discount rate $)$ SavingsYear2 $+1 /(1+$ discount rate $) 2+$ Savings Year3 / (1 + discount rate) 3

NPV $=1508961 /(1+0.1) 1+465430 /(1+0.1) 2+451150(1+0.1) 3-2320000=503,508.05$

Since NPV of the project has been positive and has NPV is the best method of assessing whether a project should be elected or not, the company should choose project.

\section{Conclusions}

1. Analysis of the costs and benefits of data center transition to PRIMO Communications in AWS shows positive financial results.

2. A related conclusion mastered control over broadband Internet that is the basis for communication of these data centers geographically dispersed and relative fragility of its architecture.

3. IT leaders are ever involved and pursuing various initiatives for energy saving in IT infrastructure and operation in any IT team

4. Cloud computing providers are more inclined to increase the efficiency of energy use for all this translates into increased profit margins

5. Industrialization and development of this new part of the information technology industry that will affect the growth of the efficiency of energy use continuously

6. Reducing dependence staff, users of computational resources from IT staff-

7. Reducing the time of development and the commercialization of new products

8. Adopting cloud technology will help mitigate the costs and risks at the same time. 


\section{Recommendations}

- A change in legislation regarding the Carbon Scheme Movement and the entry into force of tax reliefs to companies that adhere to the Code of Ethics of the EU will affect the results of the financial analysis in the financial and environmental perspective.

- Another issue on which research should be directed next is you 're creating dependency majority of European firms to

- $\quad$ those centered in the U.S. as Google or Microsoft.

- The potential benefits of Green IT mainly in energy saving should make IT managers to look at ways to increase the efficiency of operations

- The engineering staff productivity will increase due to a higher efficiency of systems and time management as well as the best of them, all of them follow the life cycle of a business with structured and productive.

- The aim of this analytical approach is to give a clearer picture, simple concrete businesses who fear making that choice, the adoption of this technology or to continue in the traditional way .

\section{References}

www.swpartners.com/documents/economistitsurvey20081025.pdf Siegel L. Let it rise, nje raport special mbi korpooratat IT publikuar ne 2008 ne The Economist http://en.wikipedia.org/wiki/Stern_Review

Stern, raport per Impaktin Ekonomik te ndryshimeve klimaterike Bowen, The economist of climate change http://www.gartner.com /technology/research.jsp

Raporti, Speed fire http://www.kpmg.com/IN/en/IssuesAndlnsights/ThoughtLeadership/The_Cloud_Changing_the_Business_Ecosystem .pdf

The cloud changing the business Ecoszstem- KPMG www.PRIMO Communications.al

Intranet.PRIMO Communications.al

http://en.wikipedia.org/wiki/Magic_Quadrant

http://en.wikipedia.org/wiki/Cloud_computing

Mell, P. \& Grance, T., 2009. The NIST Definition of Cloud Computing v15. Available www.csrc.nist.gov/groups/SNS/cloud-computing/

Armbrust, M. et al., 2009. Above the clouds: Nje veshtrim I pergjitheshem mbi cloud computing

Vaquero, L.M. et al., 2008. ACM SIGCOMM Computer Communication Review, 39(1), 50-55

McKinsey\&Company, 2009. Clearing the air on cloud computing. Gjendet ne www.google.fr/url?sa=t\&source=web\&ct=res\&cd=2\&ved $=0 \mathrm{CCAQFjAB}$

http://www.aspeninstitute.org/sites/default/files/content/docs/pubs//dentity_in_the_Age_of_Cloud_Computing.pdf

http://www.gartner.com/id=977217

http://www.forrester.com/How+Secure+Is+Your+Cloud/fulltext/-/E-RES45778

www.forrester.com

http://www.forrester.com/Is+Green+IT+Your+Emperor+With+No+Clothes/fulltext/-IE-RES46597?docid=46597

http://www.forrester.com/Christopher-Mines 